\title{
Das sechste Staatsorgan - eine Debatte ohne Folgen?
}

Es geschieht nicht oft, dass man sich nach zwei Jahrzehnten noch an innenpolitische Debatten, schon gar parteipolitische erinnert. Eine solche Diskussion löste der damalige Bundespräsident Richard von Weizsäcker in einem Gesprächsbuch mit den ZEIT-Journalisten Gunter Hofmann und Werner A. Perger im Juni 1992 aus. Weil er eine Diskussion über den Zustand unserer Demokratie initiieren wollte, nahm er dort zu Fragen der deutschen Einheit, zur Außen- und Europapolitik und zum „Parteienstaat oder Die Zukunft der liberalen Demokratie" (Hofmann/Perger 1992: 135) Stellung. Während die beiden ersten Themen kurz nach der Wiedervereinigung gleichsam auf der Hand lagen, stach er mit seinen Bemerkungen über die Parteien in ein Wespennest, damals für den Bundespräsidenten ein ungewohntes Unterfangen. Die Wellen schlugen hoch, so hoch, dass selbst Bundeskanzler Helmut Kohl sich öffentlich äußerte und schützend vor die Parteien stellte.

Doch was ist nach all dieser Zeit aus den Anmerkungen und den wenigen Anregungen von damals geworden. Der Bundespräsident beklagte sich unter anderem, dass neben den „,ünf Verfassungsorganen des Grundgesetzes“, nämlich dem Bundestag und dem Bundesrat sowie dem Bundespräsidenten, der Bundesregierung und dem Bundesverfassungsgericht, die ,sich im Großen und Ganzen gut bewährt“ hätten, ein Zentrum entstanden sei, „welches gar nicht zu den Verfassungsorganen zählt, aber praktisch über ihnen steht“ (ebd.: 139). Mit diesem „sechsten Zentrum“ meinte Richard von Weizsäcker die „Zentralen der politischen Parteien“. Vorsichtshalber weist er zwar auf die Unverzichtbarkeit der Parteien hin, beklagt aber ihren, zum „Teil völlig beherrschenden Einfluss“ (ebd.: 140).

Diese Kritik war deutlich. Gefährlich wurde es aber für die Parteien erst dann, als der Bundespräsident beklagte, dass jedes Verfassungsorgan sich nach klaren Verfassungsrichtlinien orientieren müsse, damit würden Ausmaß und Kontrolle ihrer Machtbefugnisse bestimmt. Jedoch: ,[V]ergleichbare Vorschriften gibt es aber für die mächtigste Institution in unserem staatlich-gesellschaftlichen Leben, nämlich für die Parteien, nicht.“ (ebd.: 140). Es fehlt also an der demokratischen Kontrolle der Parteien, ein unter Legitimationsgesichtspunkten unhaltbarer Zustand. Statt aber über den Weg zu mehr Aufgabenklarheit und Machtkontrolle der Parteien zu dis- 
kutieren, entwickelte sich damals eher eine Stildebatte, vor allem über die vom Bundespräsidenten entlehnte Beschreibung der Parteien als „machtversessen und machtvergessen“ (ebd.: 164). Auch der Hinweis, die Pflichten der Parteien seien nur ,dürftig“ (ebd.: 141) festgelegt, führte zu keiner breiten Diskussion.

Neben einigen Bemerkungen zur damaligen Verfassungskommission sowie der Lage von Demokratie und Parteien in Großbritannien und den USA beklagte Richard von Weizsäcker eine „Vorteilsaufteilung zwischen Politik und Gesellschaft“ (ebd.: 167). Er meinte damit ein stilles Bündnis, in dem die Parteien den Wohlstand der Gesellschaft unangetastet lassen, um dadurch ihren Machterhalt zu sichern. Deshalb gebe es zu wenig „konzeptionelle politische Arbeit“ und „ein hohes Maß an übervernetzter Verrechtlichung der Verhältnisse und an Immobilität" (ebd.: 165). Als Lösung forderte er die gesellschaftlichen Gruppen auf, ,für die Angelegenheiten des ganzen Landes“ mitzudenken, da die Parteien dazu nur ,sehr bedingt fähig“ seien (ebd.: 166). Auch in diesem Punkt ist wenig geschehen, vielleicht sogar ein kontinuierlicher Rückgang des bürgerschaftlichen Engagements zu beklagen.

Warum hat eine solche Initiative unseres Staatsoberhauptes so wenig Folgen gezeigt? Der Grund liegt wohl darin, dass der Bundespräsident, wie es seines Amtes ist, nur Hinweise gegeben hat. Sie sind nicht aufgenommen worden. Damals fühlten sich viele in den Parteien noch sehr sicher. Sie hatten auch Großes geleistet. Die Wiedervereinigung Deutschlands und Europas, die soziale Marktwirtschaft, der Rechtsstaat und die Bürgerrechte - Deutschland war aus den Trümmern auferstanden in Einigkeit und Recht und Freiheit.

Heute ist die Lage anders: die Zukunft Europas, Deutschlands Rolle in der Welt, die neue Weltordnung, die Energiewende, der demografische Wandel und die digitale Revolution erfordern neue Antworten und große Anstrengungen. Und vor allem das Ansehen der Parteien ist gesunken. Die Parteien haben jedenfalls kein Monopol auf die Mitwirkung bei der politischen Willensbildung des Volkes mehr. Dieses war bei der Verabschiedung des Grundgesetzes auch nicht vorgesehen (Hennis 1992: 27 ff.). In den letzten Jahrzehnten schien es aber so, als ob die Parteien sich dieses erobert hätten.

Je deutlicher wird, dass die Parteien ihre vom Grundgesetz übertragene Aufgabe, an der Willensbildung des Volkes mitzuwirken, mehr schlecht als recht erfüllen, verlieren sie seit langem und immer weiter ihren Einfluss und ihre monopolartige Stellung. Diese Entwicklung ist nicht beklagenswert. Sie stellt vielmehr eine Rückkehr zur ursprünglich von den Verfassungsvätern und -müttern gewollten Normalität dar. Diese wollten, dass Parteien und Zivilgesellschaft gleichrangig Verantwortung für die Vermittlung und Gestaltung von Politik wahrnehmen, um den Bürgern die Partizipation/Teilhabe am demokratischen Leben möglich zu machen. 
Die Parteien haben sich mit zunehmender staatlicher Macht immer mehr aus der Zivilgesellschaft zurückgezogen. Sie igelten sich in den staatlichen Institutionen ein. Die Folge ist ein tiefer Vertrauensverlust und ein schlechtes Ansehen. Weil die Bürger gleichzeitig immer weniger Verantwortung für das Allgemeinwohl übernehmen und die Parteien zum Sündenbock für alles und jedes machen, besteht eine reale Gefahr für Rechtstaat und Demokratie.

Die moderne Gesellschaft ist fragmentierter geworden, das heißt gespalten. Sie orientiert sich an Individuen und nicht an Schichten, Volksgruppen und Milieus. Alles ist möglich. Man nimmt's, wie es passt. Materialismus und Bindungslosigkeit, das heißt Relativismus prägen unsere Zeit. Abhandengekommen sind mehr und mehr Wertegebundenheit, Kompromissbereitschaft sowie Amtsautorität. Das ist dann schlecht, wenn sich die Bindekräfte einer Gesellschaft und eines Staates im Rahmen dieser Entwicklung auflösen.

Die Parteien erleben dies als Abwärtsspirale mit sich verstärkenden Effekten. Weniger Mitglieder führen zu weniger direkten Kontakten in die Bevölkerung. Urteilsfähigkeit und Erfahrungswissen nehmen in der Folge ab. Mehr Medialisierung der Politik führt zum Verlust der Unmittelbarkeit der Kommunikation. Es entsteht Gesprächsunlust und mehr Inszenierungslust. Weniger Amtsautorität führt zum Rückzug aus den politischen Debatten, weil man sich zu vielen Angriffen ausgesetzt fühlt. Mehr Spezialistentum verdrängt die Fachleute für das Allgemeinwohl. Der Rückzug in die eigene Wirklichkeit führt zu gefühlter Allzuständigkeit. Die politische Wirklichkeit wird aus der Sicht des Bürgers zur gefühlten Unwirklichkeit.

Der Bundespräsident hat vor 20 Jahren den Finger in eine Wunde gelegt. Geschehen ist seitdem zu wenig, um das Problem zu lösen. Deutschland braucht eine Neuvermessung der Verantwortungsräume zwischen Staat und Gesellschaft. Die Rede von der „Parteiverdrossenheit“ reicht eben nicht aus, um die notwendigen Veränderungen einzuleiten. Die herkömmlichen Milieu-Theorien, die neoliberale Ablehnung staatlichen Handelns oder die Gegenthese der Postdemokratie eröffnen keine Wege aus der Parteienkrise. Auch die Forderung nach mehr unmittelbarer Demokratie löst das Problem nicht, da mehr Volksbeteiligung die repräsentative Demokratie nicht ersetzen kann. Die Fehler bei anderen zu suchen, bei den Medien, den Intellektuellen, den Banken, bei Europa etc., ist ebenfalls nicht weiterführend. Zwar muss eine Demokratie nicht Parteiendemokratie sein. Aber eine freiheitliche Demokratie braucht freie Parteien.

Es gibt inzwischen zudem einen Vertrauensverlust, der nicht nur die Parteien und die Politik, sondern auch die gesellschaftlichen Institutionen wie die Gewerkschaften, die Medien, die Kirchen, die Banken erreicht hat (Lammert 2012: 4). Die These 
„[d]ie gegenwärtige Schwächephase der Demokratie hat ihren Grund vor allem im Versagen der Parteien“" geht aber zu weit (Klein 2011).

Eine Demokratie muss wie jede andere Staatsform verschiedene Voraussetzungen erfüllen. Die Bürger müssen zum Beispiel die Möglichkeit haben zu bestimmen und mitzubestimmen. Wer sich fremdbestimmt fühlt, verliert das Vertrauen in die Demokratie. Die Freiheit ist mithin für die Demokratie ein zentraler Grundwert. Die Voraussetzungen menschenfreundlicher Lebensbedingungen müssen gewährleistet sein. Die Gesellschaft darf nicht gespalten sein in Arm und Reich, in Jung und Alt, in Einheimische und Zugewanderte usw. Neben der Absicherung der großen Lebensrisiken - wie Alter, Krankheit, Pflegebedürftigkeit und Arbeitslosigkeit - müssen die Bürger auch die Chance zum sozialen Aufstieg haben. Wenn das Aufstiegsversprechen nicht mehr gilt, reißt das Band zwischen den Bürgern, vor allem den jungen Bürgern, und dem Staat. Der Staat muss in einer unsicheren Welt innere und äußere Sicherheit gewährleisten.

Viele glauben heute, dass diese Grundbedingungen nicht mehr gegeben sind. Sie empfinden dies als Krise der Demokratie. Es ist eine Krise, die sich im Verborgenen entwickelt. Staat und Gesellschaft funktionieren zwar. Aber die Grundlagen des demokratischen Zusammenlebens erodieren (Schulze 1996: 33 ff.).

Die Parteien, die nach außen ein Monopol für die politische Willensbildung in Anspruch zu nehmen scheinen, leiden darunter am meisten. Aber auch die gesellschaftlichen Institutionen tragen eine Mitverantwortung für das Allgemeinwohl. Da sie aber immer häufiger als Interessenwahrer auftreten, verlieren auch sie an Vertrauen.

Nicht nur die Parteien, sondern alle staatlichen und gesellschaftlichen Institutionen müssen ihren Beitrag leisten, um Vertrauen zurückzugewinnen. Dies wird nur gelingen, wenn die Parteien ihre Aufgaben in Staat und Gesellschaft überprüfen und neu definieren. Die Parteien müssen sich zurückziehen, um Platz zu schaffen für die Bürger, die sich für ihre Interessen, aber auch für das Allgemeinwohl einsetzen. Die Bürger brauchen neue Gestaltungsmöglichkeiten in und außerhalb der Parteien.

Die Diskrepanz zwischen dem Anspruch der Demokratie und der erlebten Wirklichkeit im Handeln der Politik muss geschlossen werden (Gross 2012: 74). Peter Glotz sagt dazu: „Die Leute merken langsam, dass immer mehr Politiker die Wirklichkeit eher durch Kommentare begleiten, als sie zu beeinflussen." (Glotz 1992: 173). Noch nicht einmal die politische Agenda wird heute noch in erster Linie von den Parteien bestimmt (Veen 1992: 159). Die Macht der Parteien muss zudem besser kontrolliert werden, nicht nur durch Wahl, Medien und Verfassungsgerichte. 
Die Volksparteien von damals kämpfen heute um ihr Überleben als gestaltende Kräfte. Schwache Volksparteien beeinträchtigen die Stabilität unserer Demokratie (Korte 2009: 111).

Je mehr Parteien notwendig sind, um regierungsfähige Mehrheiten in den Parlamenten möglich zu machen, desto mehr verliert die parlamentarische Demokratie an Gestaltungskraft und Ansehen. Es ist an der Zeit über die Zukunft unseres Parteiensystems neu nachzudenken. Das deutsche Parteiensystem entspricht nicht mehr dem Lebensgefühl der Bürger. Die Fragen des Bundespräsidenten warten seit 20 Jahren auf eine überzeugende Antwort.

\section{Literatur}

Glotz, Peter, 1992: Entscheidungsteilung. Gegen das Hindenburg-Syndrom der deutschen Politik, in: Gunter Hofmann/Werner A. Perger (Hrsg.), Die Kontroverse. Weizsäckers Parteienkritik in der Diskussion, Frankfurt a. M., 170-176.

Gross, Andreas, 2012: „Mehr Demokratie wagen“, in: Cicero. Magazin für politische Kultur 12/2012.

Hennis, Wilhelm, 1992: Der „Parteienstaat“ des Grundgesetzes: Eine gelungene Erfindung, in: Gunter Hofmann/Werner A. Perger (Hrsg.), Die Kontroverse. Weizsäckers Parteienkritik in der Diskussion, Frankfurt a. M., 25-50.

Hofmann, Gunter/Perger, Werner A., 1992: Richard von Weizsäcker im Gespräch, Frankfurt a. M.

Klein, Hans H., 2011: Metamorphose der Demokratie, Frankfurter Allgemeine Zeitung, 29.8.2011.

Korte, Karl-Rudolf, 2009: Die neue Qualität des Parteienwettbewerbs, in: Jürgen Rüttgers (Hrsg.), Berlin ist nicht Weimar. Zur Zukunft der Volksparteien, Essen, 109-114.

Lammert, Norbert, 2012: Zukunft der Demokratie - Demokratie der Zukunft, in: Konrad-Adenauer-Stiftung (Hrsg.), Die Politische Meinung 5/2012, Sankt Augustin, 1-6.

Rüttgers, Jürgen, 1993: Dinosaurier der Demokratie. Wege aus der Parteienkrise und Politikverdrossenheit, Hamburg.

Schulze, Gerhard, 1996: Die Wahrnehmungsblockade - Vom Verlust der Spürbarkeit der Demokratie, in: Werner Weidenfeld (Hrsg.): Demokratie am Wendepunkt. Die demokratische Frage als Projekt des 21. Jahrhunderts, Berlin, 33-51. 
Veen, Hans-Joachim, 1992: Illusionen der Bürgergesellschaft - Die Volksparteien in der Integrationskrise, in: Gunter Hofmann/Werner A. Perger (Hrsg.), Die Kontroverse. Weizsäckers Parteienkritik in der Diskussion, Frankfurt a. M., 153-162.

Korrespondenzanschrift:

Büro Ministerpräsident a. D.

Dr. Jürgen Rüttgers

Hauptstraße 11

50226 Frechen

E-Mail: juergen.ruettgers@web.de 